\title{
Design of Pressure Monitoring and Analysis System Based on Nano-Imprint
}

\author{
Zhitao Jiang (Corresponding author) \\ School of Mechanical Engineering, Robotics Institute, Shanghai Jiaotong University \\ 800 Dongchuan Road, Shanghai200240, China \\ Tel: 86-158-2149-7525Ｅ-mail: robinjzt2010@gmail.com
}

Pingkuan Liu

School of Mechanical Engineering, Robotics Institute, Shanghai Jiaotong University

800 Dongchuan Road, Shanghai200240, China

Zhijie Wen

School of Mechanical Engineering, Robotics Institute, Shanghai Jiaotong University

800 Dongchuan Road, Shanghai200240, China

The research is financed by 863project. No. 2006AA04Z334 (Six-dimensional micro-displacement Force-wide feedback nanoimprint equipment and application)

\begin{abstract}
Aiming at the weakness and diversity of the signal of nano-imprinting pressure and to meet the requirements of detection of IPC, a signal conditioning system and software was designed based on Max 1452.The system achieved data exchange between IPC and conditioning circuit through RS232.Using data acquisition card to receive and control data acquisition circuit.The data chart was showed on the user interface based on $\mathrm{VC}++$.Experiment showed that the system not only had comparatively high linearity,but was also easy to use.
\end{abstract}

Keywords: Max1452, VC++, RS232

\section{Introduction}

Nanoimprint technology plays an important part in nanometer manufacturing and the Real-time monitoring of pressure is the most significant aspect in nano-imprint lithography technology. if the pressure is too small, then the graphics transfer is not clear; if the pressure is too large, it may damage the template or substrate. it may cause serious template broken. this issue is especially important when using more expensive electron beam lithography templates therefore,the real-time pressure detection should be reliable and accurate during the imprinting process in order to achieve accurate control of the graphics and prevent the destruction of the template and substrate.

As the stamping process requires force measurement only from $\mathrm{Z}$-axis and mainly focus on pressure measurement. Therefore, we use uniaxial tensile pressure sensor as a measurement element. In this system, we use four force sensors in order to ensure that a greater tilt of table surface will not occur. Four sensors are installed along the circumference of the table symmetrically. this method not only resolves the issue of an easily tilt table surface but also increases the sensor detection range to four times compared with a single one. The pressure head of four sensors are connected with the table using flexible hinge so as to conpensate the height difference of the four sets of sensors.

The output signal of pressure sensors is too weak and contains noise signals, so it needs filtering and amplification so as to meet the requirements of the interface of an data acquisition card. The signal conditioning circuit is designed based on Max 1452, so that when the pressure is between $0-60 \mathrm{~N}$, the range of the output of the conditioning circuit is $0-5 \mathrm{~V}$.

At present, there are many methods which are used to filter and enlarge weak signals, a much used way is to 
utilize precise op amp and active or passive filter circuit.

However, when the temperature of the workplace changed, the output of the system will involve a temperature drift error, which is a significant drawback of this kind of circuit, the greater the temperature change, the greater the error, in order to reduce this error, a compensation circuit needs to be designed, this often leads to the complexity of the design of the system. For this reason, a signal conditioning circuit and the corresponding software are designed based on Max1452.Experiment shows that this system could well meet the needs of the detection of the pressure of nanoimprint.

\section{Overall System Block Diagram}

Max1452 is a highly integrated analog-sensor signal processor optimized for industrial and process control presure sensors that utilize piezo-resistive bridge Transducers. The max 1452 provides amplification, calibration and temperature compensation that enables an overall performance approaching the inherent linearity of the sensor. The fully analog signal path introduces no quantization noise in the output signal while enabling digitally controlled trimming with the integrated 16-bit DACs within $\pm .02 \%$.

The max 1452 architecture includes a programmable sensor excitation, a 4-bit programmable-gain amplifier, output signal clip-level diagnostics, a 768-byte interal EEPROM, four 16-bit DACs, and an on-chip temperature sensor. In addition to Offset and Span compensation, the max 1452 provides a unique temperature compensation strategy for Offset TC and FSO TC thar was developed to provide a remarkable degree of flexibility while minimizing testing costs.

The customer can select from 1 to 144 temperature points to compensate their sensor. This allows the latitude to compensate a sensor with a simple first order linear correction or match an unusual temperature curve. By proggramming up to 114 independent 16-bit EEPROM locations to correct performance in $1.5^{\circ} \mathrm{C}$ temperature increments over a range of $-40^{\circ} \mathrm{C}$ to $125^{\circ} \mathrm{C}$. The Max 1452 (Figure 1) provides an analog amplification path for the sensor signal. An input referred offset summing junction allows for a digitally generated voltage signal to be added to the transducer signal. The amplified and combined transducer offset and IRO DAC signal is summed with two independent offset adjustment voltages, both offset adjustment voltages are derived from 16-bit DACs called Offset DAC and Offset Temperature Coefficient DAC(OTC DAC). Signal conditioning circuit based on Max1452 is installed in the stamping head, the signal conditioning circuit installed is shown in Figure 2.

Max1452 is a programmable chip, including internal amplifier, EEPROM and four 16-bit DAC which need to be programed to set the specific value before being used, Max1452 provides a single pin(DIO) to communicate with the host computer through RS232 interface, the DIO serial interface is used for asynchrous serial data communications between the Max1452 and a host computer9(Fig. 3). The Max1452 will automatically detect the baud rate of the computer when the host transmits the initizlization sequence. Data formats is always 1 start bit, 8 data bits, 1 stop bit and no parity.

\section{The design of the interface between Max1452 and the computer}

In my design, Max6105 is used to power the system, the system is made up of four independent signal conditioning circuit, in order to reduce interference and meet the current demand of each channel, each channel is powered independently(Fig. 4).

Signal conditioning and temperature compensation are achieved through the circuit of Max1452, there are four force sensors installed in the system, so the signal conditioning circuit contains four channels, single channel signal conditioning circuit is shown in Figure 5.

Max1452 normally operates in analog mode, if you want to program the chip, it must be changed to digital mode.Max 1452 utilizes asynchronous serial communication to realize data exchange with the outside. An interface circuit(Fig. 6) is also designed based on RS232 to translate voltage levels between the PC and the circuit.

\section{The implementation of the communication protocol}

Commonly used communication sequence include initialization sequence, reinitialization sequence, serial interface command format, special command sequences. Sending the initialization sequence shown below enables the Max1452 to establish the baud rate that initializes the serial port. The initialization sequence is one byte transmission of $01 \mathrm{hex}$, as follows. The first start

\section{1}


bit 0 initiates the baud rate synchronization sequence. The 8 data bits 01 hex(LSB first) follow this and then the stop bit, which is indicated above as a 1 , terminates the baud rate synchronization sequence.

The Max1452 allows for relearning the baud rate. The reinitialization sequence is one byte transmission of FFhex, as follows. When a serial reinitialization sequence is received, the receive logic resets itself ot its power-up state and waits for the initialization sequence. The initialization sequence must follow the reinitialization sequence in order to re-establish the baud rate.

\section{1}

All communication commands into the Max1452 follow a defined format utilizing an interface register set(ISR).The ISR is an 8-bit command that contains both an interface register set data(ISRD) nibble(4-bit) and an interface register set address(ISRA) nibble(4-bit).All internal calibration registers and EEPROM locations are accessed for read and write through this interface register set

\section{The programming of the host}

The software of the communication interface is prepared using $\mathrm{VC}++$, the main functions of the interface include writing of the registers, online proggramming, and debugging, writing of EEPROM, gain control, and setting of the configuration registers and the selection of output port. The software interface running in the host PC is shown in Figure. 7.

Set the Max 1452 values using the user interface according to the Figure. 8, an important thing you should remember is that you must set the Max1452 in digital mode by keeping the unlock pin high before reading or writing the registers of the chip.

\section{System Calibration}

In order to accurately display real-time pressure values, the four sets of presure sensors need to be calibrated, considering the four sets of sensors are identical, only one sensor is calibrated in this system. The data of calibration are shown in Table 1, the curve of calibration is show in Figure 9.

\section{Conclusion}

In this paper, the circuit based on Max1452 is designed in order to filter, amplify, and compensate drift caused by temperature. In the presure range of $0-60 \mathrm{~N}$, the linear error of the system is relatively small and can better complete the task of real-time detection of presure.

\section{References}

ChEN Ligang, FU Jianhua \& BAI Lei. (2008). Improve the quality of the water level sensor signal using Max1452. Earthquake Research In ShanXi 135:2-4

LI Changlin, GAO. (2006). Visual C++ serial communication technology and typical example.Beijing Tsingha University Press, (chapter7).

WANG Chengjiang, WANG Anming, \& ZHANG Yuhua. (2002). Testing for Vibration in Machining of Superfine Granule. Automation\&Measurement.

WANG Pitao. (2006). Design of high-precision signal conditioning system[D]. Shangdong University,(Chapter 4).

ZHANG Hao, MENG Kaiyuan \& CAO Qingnian. (2006). Design of temperature compensation system with MSP430 and Max1452. Control\&Automation.

ZHAO Yan \& LIU Zhizhen. (2006). The temperature compensation of pressure sensor based on Max1452. Industrial Control.

ZHAO Yan, LI Yonghong \& WANG Enhuai. (2009). The calibration system for mems pressure sensor based on Max1452.Instrmment Technique and Sensor 10:1-3 
Table 1. The calibrated datas of one sensors

\begin{tabular}{|l|l|l|l|l|l|l|}
\hline presure/g & 0 & 10 & 50 & 100 & 150 & 200 \\
\hline voltage/V & 0.51 & 0.53 & 0.68 & 0.81 & 0.98 & 1.11 \\
\hline presure/g & 250 & 300 & 350 & 400 & 450 & 500 \\
\hline voltage/V & 1.28 & 1.43 & 1.55 & 1.72 & 1.88 & 2.1 \\
\hline
\end{tabular}

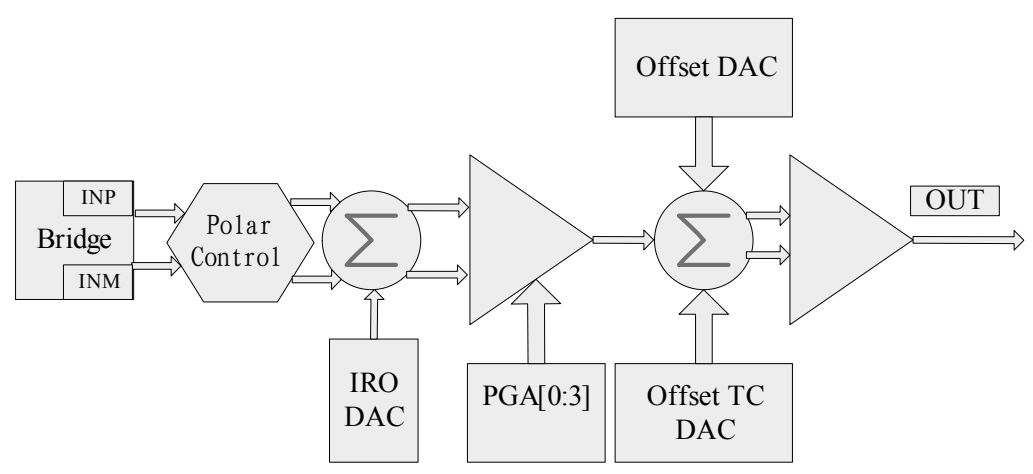

Figure 1. Analog amplifier of $\max 1452$

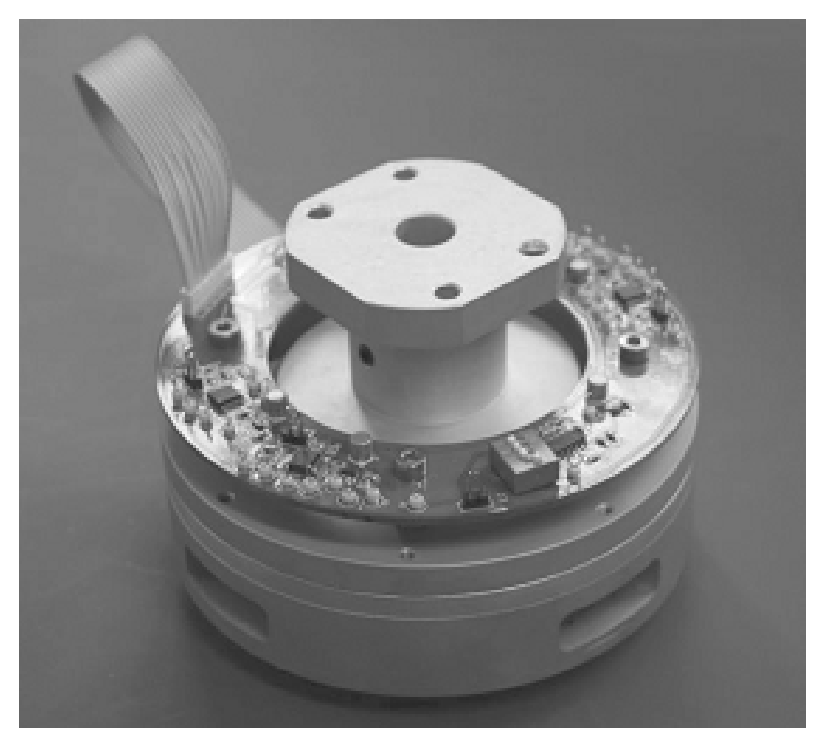

Figure 2. Imprint head and force sensor system 


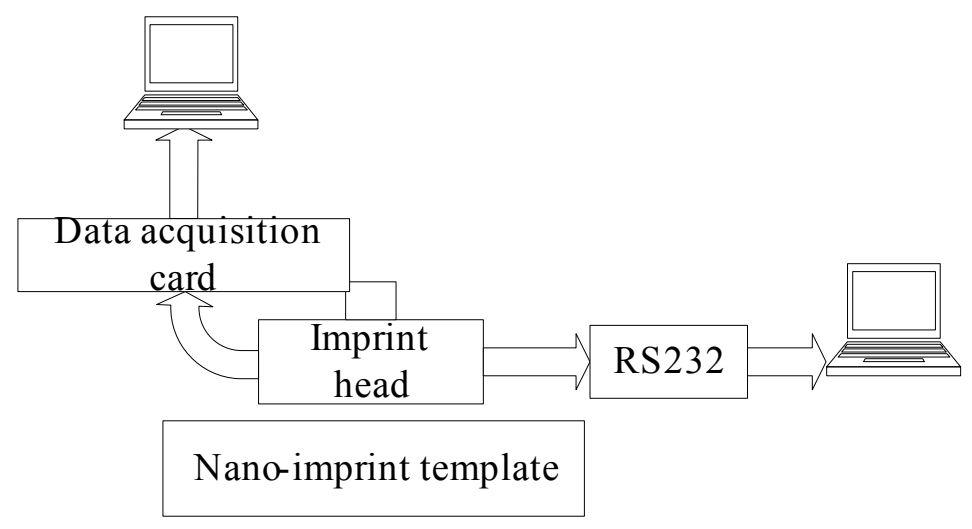

Figure 3. Structure diagram of nano-imprinting and data acquisition and analysis system

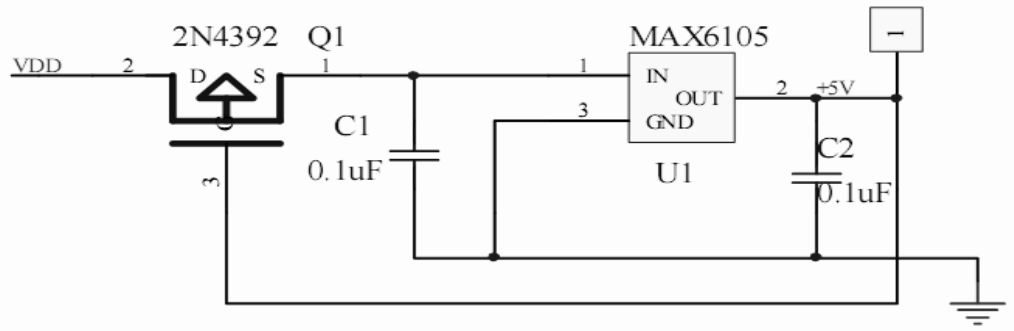

Figure 4. Single power supply circuit

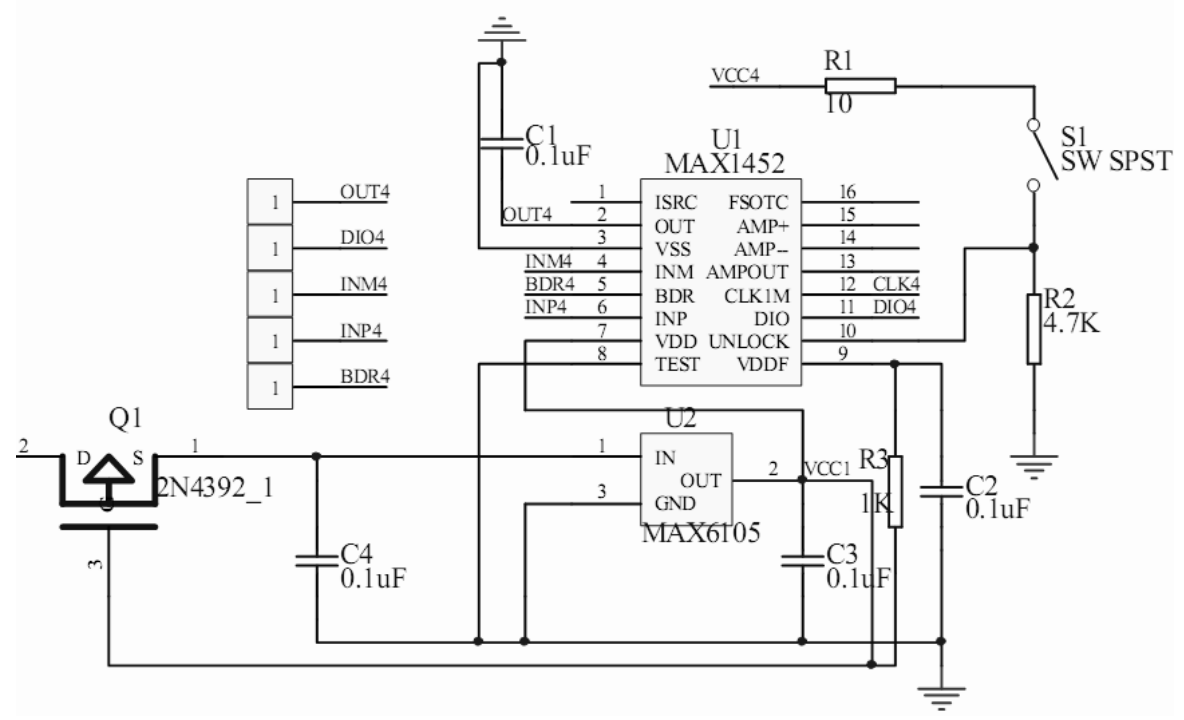

Figure 5. Max1452 signal conditioning circuit 


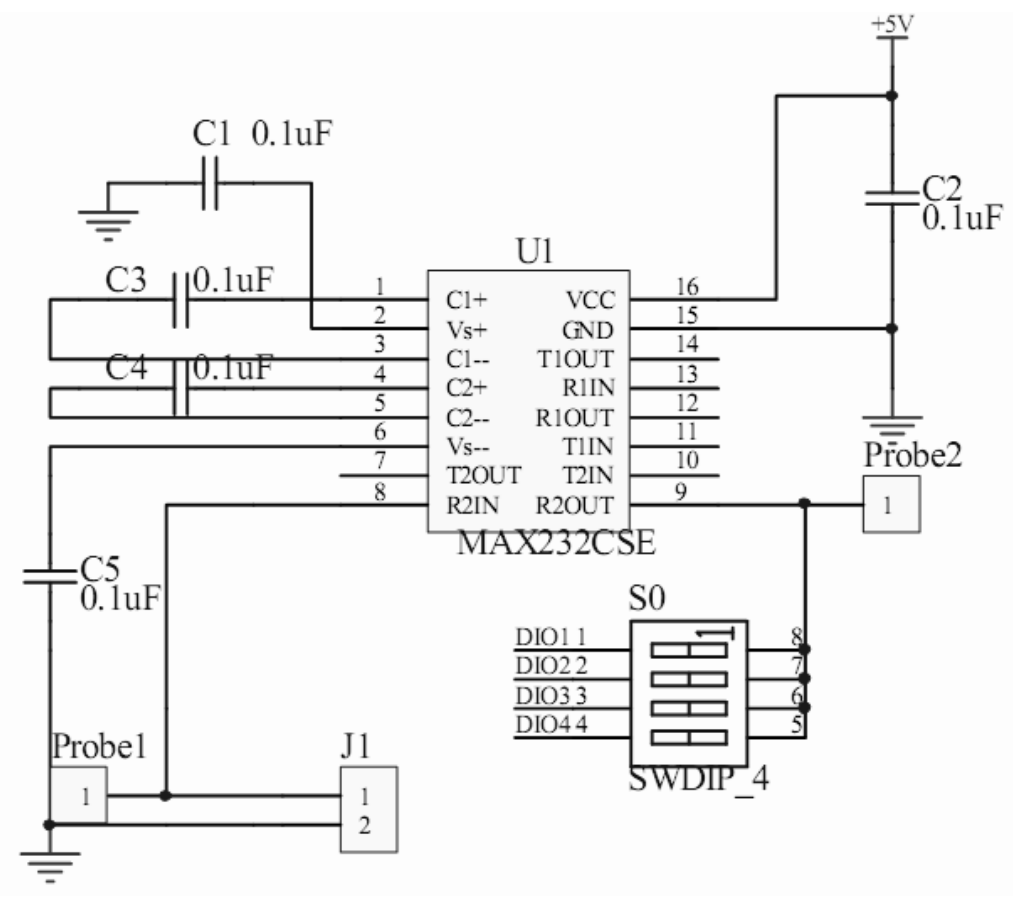

Figure 6. RS232 interface circuit

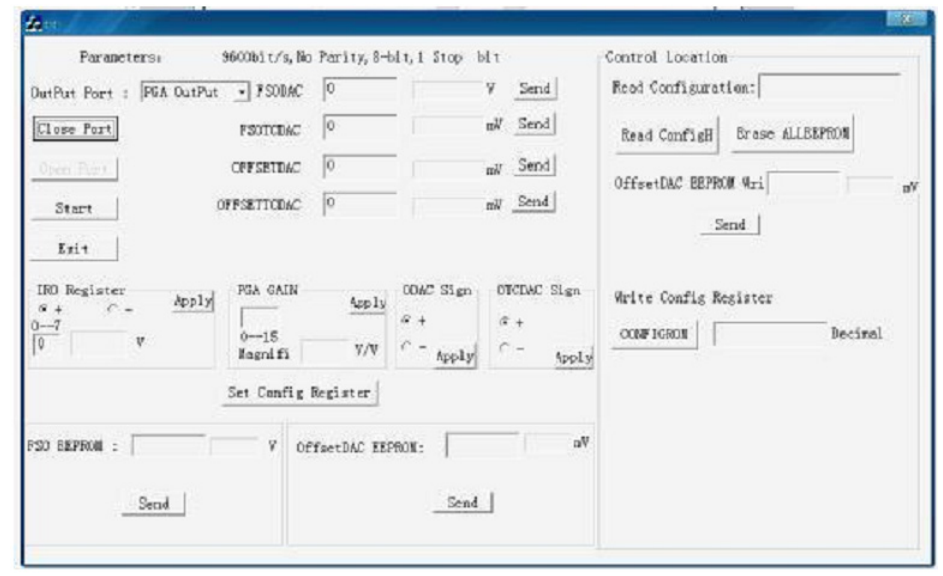

Figure 7. Max1452 Communication software interface 


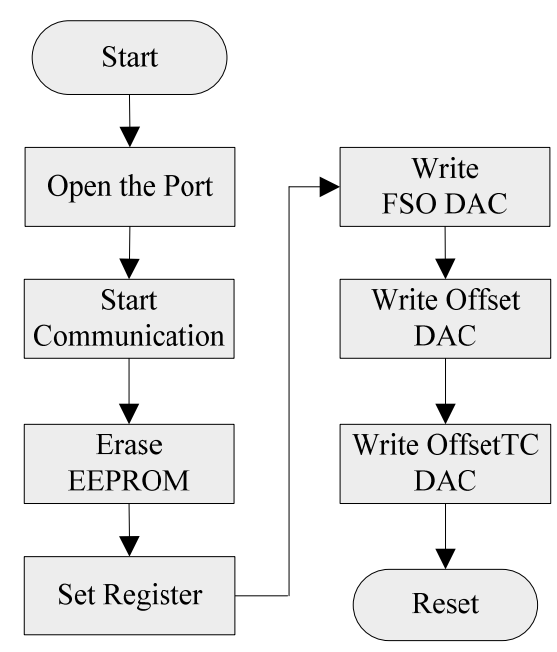

Figure 8. Max1452 system communication software flow chart

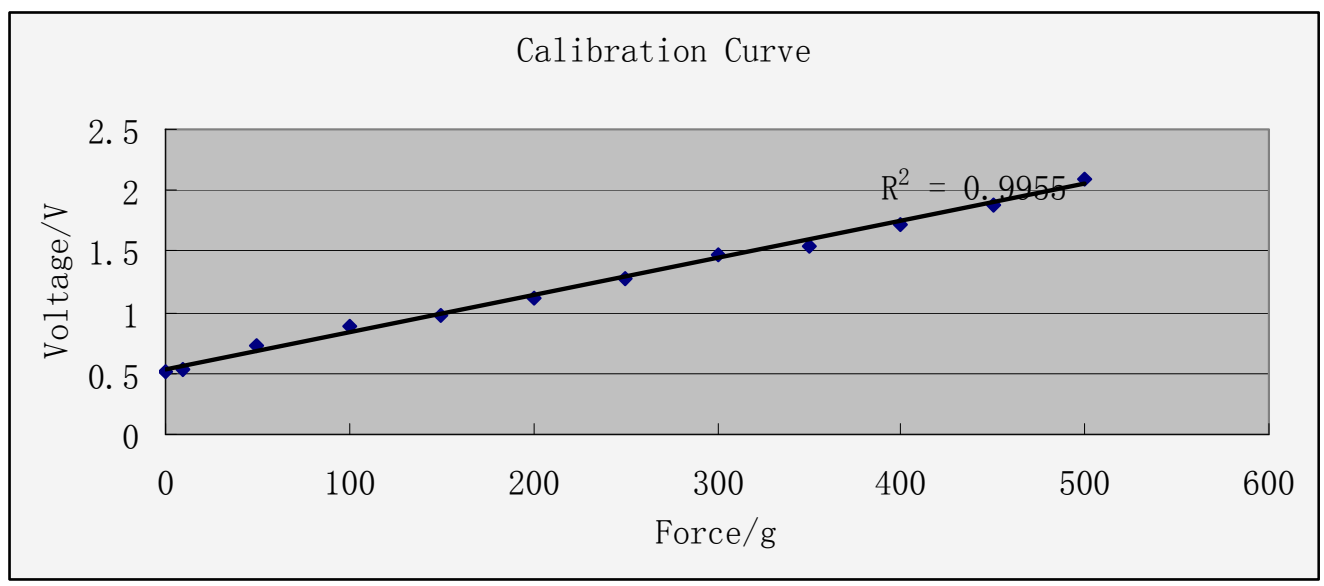

Figure 9. The curve of sensor calibration 